\title{
Variabilidad estructural de la comunidad de macrófitas en un gradiente pluviométrico en la provincia de Chubut
}

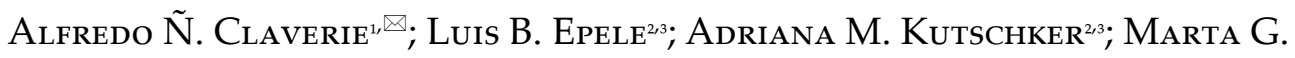 \\ GreCH $^{23} ;$ Luz M. Manzo ${ }^{2}$ \& María L. Miserendino ${ }^{2 / 3}$ \\ ${ }^{1}$ Instituto de Ciencias Polares, Ambiente y Recursos Naturales (UNTDF-CONICET). Ushuaia, Tierra del Fuego, Argentina. \\ ${ }^{2}$ Centro de Investigación Esquel de Montaña y Estepa Patagónica (CONICET). Esquel, Chubut, Argentina. ${ }^{3}$ FCNyCS- \\ Universidad Nacional de la Patagonia San Juan Bosco (CONICET-UNPSJB). Esquel, Chubut, Argentina.
}

\begin{abstract}
Resumen. Los mallines son humedales patagónicos con suelos en permanente anegación, en los cuales se pueden generar lagunas con una vasta biodiversidad. En estos ecosistemas, las plantas acuáticas (i.e. macrófitas) se desarrollan y presentan hábitos de vida para adaptarse a las condiciones de humedad, siendo capaces de regular las condiciones fisicoquímicas del agua. Una característica distintiva de la Patagonia es la disminución pluvial en un gradiente oeste-este que genera tres biozonas: bosque, ecotono y estepa. Estas biozonas fueron caracterizadas en base a vegetación terrestre, pero aún no se ha evaluado si los ensambles de macrófitas se corresponden con ellas. El objetivo de este estudio es comparar los atributos de la comunidad de macrófitas acuáticas y de las plantas terrestres asociadas a humedales presentes en las tres biozonas. Durante el verano 2013-2014 se muestrearon 20 lagunas someras de la provincia de Chubut, se analizaron las características físicas y químicas del agua y se registró la riqueza taxonómica de macrófitas. Las macrófitas fueron clasificadas según su origen y hábito, y se calculó su diversidad. Se identificaron 62 taxones, la mayoría pertenecientes a las familias Cyperaceae, Poaceae y Ranunculaceae. Las lagunas esteparias se diferenciaron de las de ecotono y de bosque por poseer $\mathrm{pH}$ alcalino y valores elevados de conductividad y de fósforo reactivo soluble (PRS). Los valores altos de PRS favorecieron la abundancia de macrófitas y macroalgas. Sin embargo, las concentraciones elevadas de fósforo total y de clorofila a fitoplanctónica indicaron que tres lagunas estarían eutrofizadas. Las macroalgas fueron más abundantes en los sitios con mayor estado trófico y la cobertura de macrófitas emergentes aumentó en esos sitios. Dado que las lagunas estudiadas funcionarían como reservorios de diversidad de macrófitas acuáticas, conocer la relación comunidad biológica-ambiente sería útil para generar planes tendientes a conservar la biodiversidad de los mallines patagónicos.
\end{abstract}

[Palabras clave: biodiversidad, mallines, humedales, lagunas someras, Patagonia]

\begin{abstract}
Structural variability of the macrophytes community as a function of a pluviometric gradient in the province of Chubut. Mallines are Patagonian wetlands characterized by permanent waterlogging on its soils, sustaining small shallow lakes that hosts a vast biodiversity. In these ecosystems, aquatic plants (i.e. macrophytes) develop different life forms adapted to humid conditions, allowing them to regulate the physical and chemical conditions of the mallín. A distinctive characteristic of Patagonia is the exponential west-east decrease in rainfall, which generates three biozones: forest, ecotone and steppe. These biozones have been typified using landform plants, but whether macrophytes assemblages are related to each biozone or not has not been evaluated yet. The aim of this work is to compare the attributes of the macrophyte and wetlands landform plants community in each biozone. During the 2013-2014 austral Summer, 20 shallow lakes were sampled in the west province of Chubut, assessing macrophyte richness and physical and chemical water variables. Macrophytes were classified according to their life forms, origin, and its diversity was calculated. A total of 62 taxa were determined, most of them corresponding to the Cyperaceae, Poaceae and Ranunculaceae families. The steppe exhibited alkaline $\mathrm{pH}$ values, and higher conductivity and soluble reactive phosphorus (SRP) than the ecotone and the forest. High SRP values favored the abundance of macrophytes and macroalgae. However, three sites were eutrophic, exhibiting high chlorophyll $a$ and total phosphorus values. Those sites showed higher abundances of macroalgae and emerging macrophytes. The sampled shallow lakes would act as reservoirs of macrophyte diversity at both local and regional scales. Thus, understanding the relationships among macrophytes and environmental variables could be useful for the mallines biodiversity conservation plans.
\end{abstract}

[Keywords: biodiversity, mallines, macrophytes, wetlands, shallow lakes, Patagonia] 


\section{INTRODUCCIÓN}

Los humedales son ecosistemas complejos que se caracterizan por presentar suelos anegados, con el nivel freático en la superficie terrestre o cerca de ella, y a veces agua superficial léntica poco profunda (Collantes and Faggi 1999). Estos ambientes se encuentran entre los ecosistemas más productivos del mundo (Mitsch and Gosselink 2015), contribuyen a regular el flujo de agua en las cuencas y constituyen reservorios de diversidad biológica y de material genético vegetal (Secretaría de la convención de Ramsar 2006). Los mallines son los humedales propios de la Región Patagónica argentina, y su nombre en lengua mapuche significa tierra pantanosa, área inundada o estero (Erize 1960). Los mallines suelen generarse en zonas aluviales, deprimidas, donde en la mayoría de los casos el horizonte impermeable puede limitar la percolación (Hauser 1984). En la Patagonia argentina, los mallines representan $\sim 5 \%$ de la región (Iriondo 1989) y se ubican desde la cordillera de los Andes hasta la costa atlántica a lo largo de un gradiente de precipitación oeste-este (3000 a $200 \mathrm{~mm}$ de media anual). Además, representan un gran valor económico para la región, ya que poseen especies vegetales palatables que constituyen excelentes fuentes de forrajeo para el ganado ovino y bovino, en especial en lugares en donde la disponibilidad de pasturas suele ser baja, como ocurre en las grandes extensiones de estepa patagónica (Raffaele 1999).

Otro factor distintivo de los humedales, y por tanto de los mallines, es la presencia de comunidades de organismos fotosintéticos adaptados a ambientes húmedos, que, en sentido amplio, se denominan macrófitas. Por lo tanto, en este trabajo el término 'macrófitas' incluye tanto a plantas acuáticas como a macroalgas. Éstas representan a los principales productores primarios en los humedales, e influyen en los ciclos geoquímicos al funcionar como vínculo entre la columna de agua y el sustrato (Thomaz and Cunha 2010). Además de estructurar las propiedades físicas y químicas de los humedales, influyen en su hidrología y su régimen de sedimentación, por ejemplo, al modificar las corrientes y la desincronización de las crecidas (Gosselink and Turner 1978). Algunos autores establecen que ciertos atributos de la comunidad de macrófitas (e.g., proporción de exóticas y nativas, riqueza y abundancia de especies) pueden evidenciar los cambios en las características físicas, químicas y biológicas de los ecosistemas (Carpenter and
Lodge 1986; Mushet et al. 2002; Balcombe et al. 2005), hecho que también fue comprobado en mallines patagónicos (Gaitán et al. 2011; Kutschker et al. 2014; Manzo et al. 2020). Dichos atributos también se podrían utilizar para detectar efectos de las variaciones pluviométricas, que también podrían alterar las propiedades físicas y químicas del agua (Junk et al. 1989; Thomaz et al. 1992; Chimner et al. 2011).

Considerando la sensibilidad de los humedales patagónicos en el actual contexto de cambio climático (Perotti et al. 2005; Crego et al. 2013; Epele et al. 2021), es fundamental entender las variaciones regionales de la biodiversidad de macrófitas para fomentar un aprovechamiento sustentable de los mallines y contribuir con su conservación. En este sentido, el presente estudio pretende evaluar y relacionar las características abióticas y bióticas (i.e., macrófitas acuáticas y plantas terrestres asociadas a humedales) de lagunas someras de mallines, las cuales se distribuyen en tres biozonas de la provincia de Chubut. En base a estudios previos realizados en la estepa y los bosques patagónicos (San Martin 2013; Macchi 2017), hipotetizamos que la diversidad de macrófitas es menor en la estepa que en el bosque. Entender las características emergentes de estos hidrosistemas contribuirá a generar herramientas teóricas y empíricas para cuidarlos y manejarlos de manera sustentable.

\section{Materiales y Métodos}

\section{Área de estudio}

La provincia de Chubut se encuentra en el centro-norte de la Región Patagónica (Figura 1) y ocupa un territorio de $224686 \mathrm{~km}^{2}$. El clima de esta región es templado-frío y las precipitaciones decrecen significativamente desde los Andes patagónicos hacia la estepa (Paruelo et al. 1998). Esta cordillera genera una barrera que retiene la mayor parte de la humedad proveniente del Océano Pacífico en su porción occidental, y termina por secar estas masas en su parte oriental y hacia el resto de la Región Patagónica. Estas masas de aire provenientes del este de los Andes generan un marcado gradiente oeste-este en el régimen hídrico (Paruelo et al. 2004). En el extremo más occidental, cercano al límite con Chile, se encuentran los Bosques Subantárticos, con precipitaciones que oscilan entre 800 y 3000 mm anuales, y masas boscosas compuestas principalmente por árboles del género 


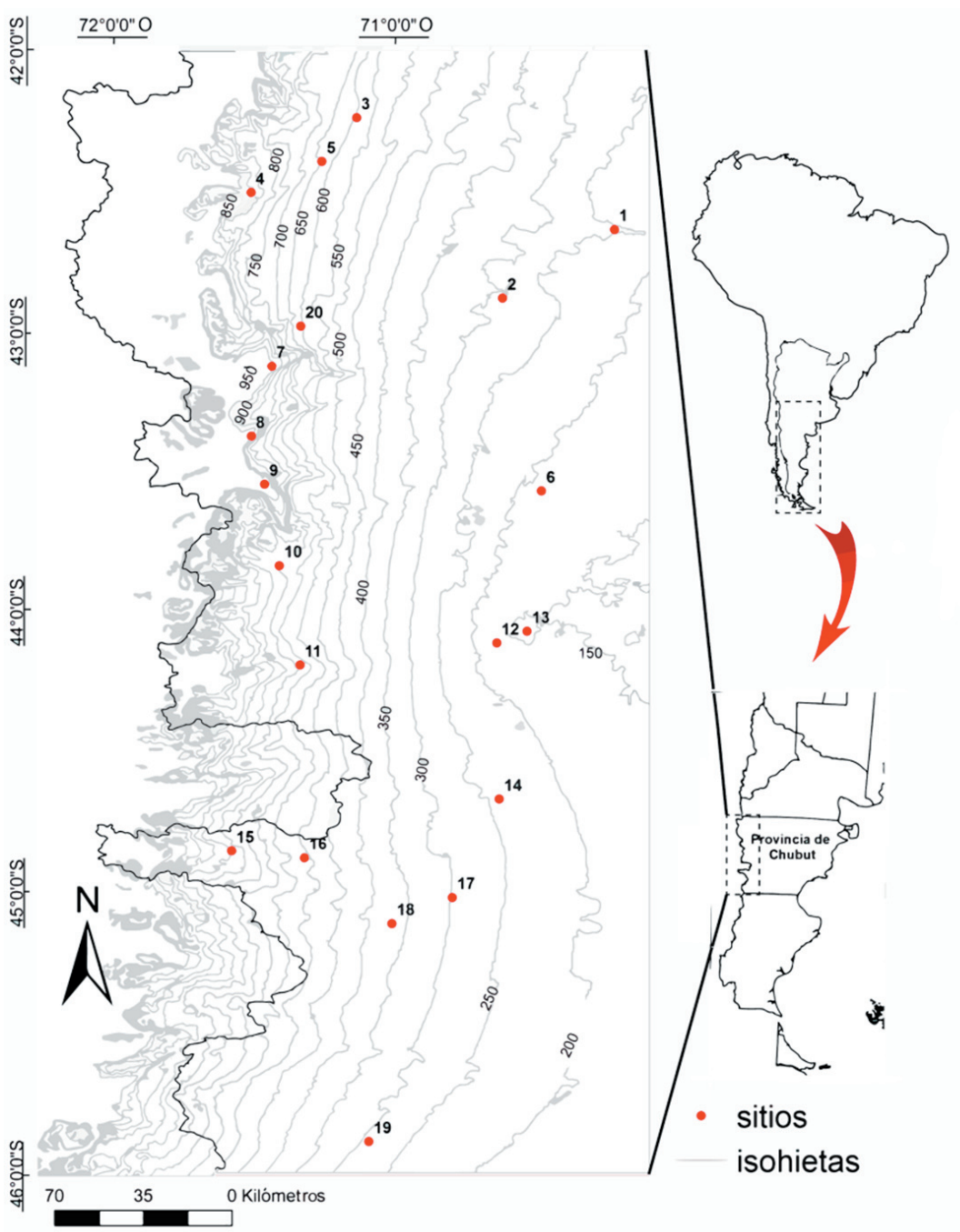

Figura 1. Mapa de isohietas de $50 \mathrm{~mm} / a n ̃ o$ del sector oeste de la provincia de Chubut, con la ubicación de las 20 lagunas estudiadas.

Figure 1. Isohyet map of $50 \mathrm{~mm} /$ year of the west region of province of Chubut, showing the location of the 20 studied shallow lakes.
Nothofagus. Las precipitaciones en la estepa varían entre 100 y $300 \mathrm{~mm}$ anuales, mientras que en el ecotono, el rango de variación oscila entre 300 y $700 \mathrm{~mm}$ anuales, aproximadamente (del Valle et al. 1998). La estructura de la comunidad de macrófitas en el centro-norte de la Región Patagónica se compone mayormente por dicotiledóneas, monocotiledóneas y macroalgas, siendo las familias Cyperaceae, Poaceae y Juncaceae las más representativas (Gaitán et al. 2011; Kutschker et al. 2014).

\section{Selección de sitios de muestreo}

Teniendo en cuenta la diversidad bioclimática en el área de estudio, los sitios de muestreo se localizaron cubriendo el gradiente norte-sur de la provincia de Chubut (entre los paralelos $42^{\circ}$ y $46^{\circ} \mathrm{S}$ ) y desde el bosque en el oeste hacia la estepa al este (entre los meridianos $70^{\circ}$ y $\left.71^{\circ} \mathrm{O}\right)$. Siguiendo los criterios propuestos por del Valle et al. (1998), las tres biozonas se definieron en función de la precipitación media anual: estepa ( $<300 \mathrm{~mm} / \mathrm{año})$, ecotono (entre 300 y $700 \mathrm{~mm} / \mathrm{año}$ ) y bosque (>700 $\mathrm{mm} / \mathrm{año}$ ) (Figura 1). Luego, se realizó una selección preliminar de las lagunas de mallines a muestrear, distribuyendo las mismas en las tres biozonas; así se definieron 8 lagunas de estepa, 7 de ecotono y 5 de bosque (Google Earth Pro 7.1 2013). Para definir a cuál biozona correspondía cada sitio de estudio, se trazaron curvas pluviométricas (isohietas) de $50 \mathrm{~mm} /$ año en un mapa a partir del modelo SRTM de 30 m, obtenido con Google Earth Engine, y con un layout generado en el programa Esri ArcMapTM 10.1. Sobre la base de esas imágenes se seleccionó y muestreó un total de 20 lagunas someras (Tabla 1). Las lagunas fueron muestreadas en una única ocasión entre diciembre de 2013 y enero de 2014, ya que en la región resulta el período más apropiado para estudiar tanto la composición de las macrófitas (e.g., fenología y presencia de flores), como para estimar su abundancia (Epele and Miserendino 2015). 
Tabla 1. Sitios de muestreo con sus nombres asignados en función a la localidad o ruta más cercana, biozona a la que pertenecen y ubicación geográfica (provincia de Chubut).

Table 1. Sampling sites with their assigned names based on the closest town or main route, biozone and geographical location (province of Chubut).

\begin{tabular}{|c|c|c|c|c|c|}
\hline Sitio & Nombre & Código & Biozona & Latitud & Longitud \\
\hline 1 & Piedra Parada & PPA & Estepa & $42^{\circ} 38^{\prime} 20.4^{\prime \prime} \mathrm{S}$ & $70^{\circ} 13^{\prime} 44.6^{\prime \prime} \mathrm{O}$ \\
\hline 2 & Arroyo Pescado & $\mathrm{APE}$ & Estepa & $42^{\circ} 52^{\prime} 52^{\prime \prime} \mathrm{S}$ & $70^{\circ} 37^{\prime} 33^{\prime \prime} \mathrm{O}$ \\
\hline 3 & El Maitén & MAI & Ecotono & $42^{\circ} 14^{\prime} 17.2^{\prime \prime} \mathrm{S}$ & $71^{\circ} 8^{\prime} 40.6^{\prime \prime} \mathrm{O}$ \\
\hline 4 & Cholila & $\mathrm{CHO}$ & Bosque & $42^{\circ} 30^{\prime} 15.2^{\prime \prime} \mathrm{S}$ & $71^{\circ} 31.1^{\prime} 11.5^{\prime \prime} \mathrm{O}$ \\
\hline 5 & Benetton & BTT & Ecotono & $42^{\circ} 23^{\prime} 39.5^{\prime \prime} \mathrm{S}$ & $71^{\circ} 16^{\prime} 3.9^{\prime \prime} \mathrm{O}$ \\
\hline 6 & Ruta 25 & R25 & Estepa & $43^{\circ} 34^{\prime} 6.1^{\prime \prime} \mathrm{S}$ & $70^{\circ} 29^{\prime} 15.8^{\prime \prime} \mathrm{O}$ \\
\hline 7 & Corinto & $\mathrm{COR}$ & Bosque & $43^{\circ} 7^{\prime} 25.25^{\prime \prime} \mathrm{S}$ & $71^{\circ} 26^{\prime} 44.7^{\prime \prime} \mathrm{O}$ \\
\hline 8 & Ruta 17 & $\mathrm{R} 17$ & Bosque & $43^{\circ} 22^{\prime} 23.5^{\prime \prime} \mathrm{S}$ & $71^{\circ} 31^{\prime} 6.5^{\prime \prime} \mathrm{O}$ \\
\hline 9 & Corcovado & CRC & Bosque & $43^{\circ} 32^{\prime} 10.9^{\prime \prime} \mathrm{S}$ & $71^{\circ} 28^{\prime} 17.1^{\prime \prime} \mathrm{O}$ \\
\hline 10 & Lago Guacho & LGU & Ecotono & $43^{\circ} 50^{\prime} 3.6^{\prime \prime} \mathrm{S}$ & $71^{\circ} 25^{\prime} 8.5^{\prime \prime} \mathrm{O}$ \\
\hline 11 & Río Pico & RPI & Ecotono & $44^{\circ} 11^{\prime} 18.7^{\prime \prime} \mathrm{S}$ & $71^{\circ} 20^{\prime} 45.2^{\prime \prime} \mathrm{O}$ \\
\hline 12 & Ruta 19 & R19 & Estepa & $44^{\circ} 6^{\prime} 37.1^{\prime \prime} \mathrm{S}$ & $70^{\circ} 58^{\prime} 48.6^{\prime \prime} \mathrm{O}$ \\
\hline 13 & Gobernador Costa & GBC & Estepa & $44^{\circ} 4^{\prime} 37.4^{\prime \prime} \mathrm{S}$ & $70^{\circ} 32^{\prime} 19.9^{\prime \prime} \mathrm{O}$ \\
\hline 14 & Ex ruta 40 & $\mathrm{X} 40$ & Estepa & $44^{\circ} 39^{\prime} 57.9^{\prime \prime} \mathrm{S}$ & $70^{\circ} 38^{\prime} 15.5^{\prime \prime} \mathrm{O}$ \\
\hline 15 & Lago Fontana & LFT & Bosque & $44^{\circ} 51^{\prime} 2.6^{\prime \prime} \mathrm{S}$ & $71^{\circ} 35^{\prime} 18.8^{\prime \prime} \mathrm{O}$ \\
\hline 16 & Ruta 57 & R57 & Ecotono & $44^{\circ} 52^{\prime} 30.5^{\prime \prime} \mathrm{S}$ & $71^{\circ} 19^{\prime} 49.2^{\prime \prime} \mathrm{O}$ \\
\hline 17 & Río Senguer & RSG & Estepa & $45^{\circ} 1^{\prime} 21.8^{\prime \prime} \mathrm{S}$ & $70^{\circ} 48^{\prime} 16.4^{\prime \prime} \mathrm{O}$ \\
\hline 18 & Estancia & EST & Ecotono & $45^{\circ} 6^{\prime} 35.1^{\prime \prime} \mathrm{S}$ & $71^{\circ} 1^{\prime} 28.9^{\prime \prime} \mathrm{O}$ \\
\hline 19 & Lago Blanco & LBL & Estepa & $45^{\circ} 53^{\prime} 12.4^{\prime \prime} \mathrm{S}$ & $71^{\circ} 6^{\prime} 13.6^{\prime \prime} \mathrm{O}$ \\
\hline 20 & Nahuel Pan & NHP & Ecotono & $42^{\circ} 58^{\prime} 53.2^{\prime \prime} \mathrm{S}$ & $71^{\circ} 10^{\prime} 37.1^{\prime \prime} \mathrm{O}$ \\
\hline
\end{tabular}

\section{Caracterización ambiental}

Por medio del modelo de precipitación desarrollado por Gaitán et al. (2014) se obtuvo información de la precipitación media anual (PMA) para cada sitio (en base a datos recolectados entre los años 2000 y 2011). Se midió el largo y el ancho de los espejos de agua utilizando un telémetro láser (BOSCH GLM 250 VF Professional). La profundidad media se obtuvo de promediar 5 mediciones en un perfil desde el borde del cuerpo de agua hasta el centro con una varilla graduada. Asimismo, con una sonda multiparámetro Hach Sension 156 (una medición en cada sitio) se estimaron las variables temperatura del agua $\left({ }^{\circ} \mathrm{C}\right)$, $\mathrm{pH}$, conductividad $(\mu \mathrm{S} / \mathrm{cm})$, total de sólidos disueltos $(\mathrm{mg} / \mathrm{L})$, salinidad $(\% \mathrm{o})$, concentración $\left(\mathrm{mgO}_{2} / \mathrm{L}\right)$ y porcentaje de saturación de oxígeno disuelto. En el área inundable de cada sitio se recolectó una muestra de $2 \mathrm{~L}$ de agua, la cual fue parcialmente filtrada (filtros AC $0.45 \mu \mathrm{m}$ ) y refrigerada en el campo a $4{ }^{\circ} \mathrm{C}$. Las muestras de agua fueron llevadas al laboratorio, donde se conservaron en freezer y se analizaron dentro de los 3 meses posteriores a su colecta utilizando métodos estandarizados (APHA 1992). Se determinaron las concentraciones de los principales nutrientes: nitrógeno total (NT), fósforo total (PT) (con estos dos primeros parámetros se calculó el coeficiente NT/PT), nitratos más nitritos $\left(\mathrm{NO}_{3}^{-}+\mathrm{NO}_{2}^{-}\right)$, amonio
$\left(\mathrm{NH}_{4}^{+}\right)$y fósforo reactivo soluble (PRS). Para estimar el total de sólidos en suspensión (TSS) se filtró un volumen conocido de agua en filtros previamente secados y pesados (prefiltro de fibra de vidrio 13400-47-Q, Sartorius Stedim Biotech). En laboratorio, los prefiltros con sedimento se secaron en estufa eléctrica por cuatro horas a $104{ }^{\circ} \mathrm{C}$. El valor final resultó de la diferencia entre el peso final y el inicial del filtro, medido en balanza electrónica OHAUS Explorer, $\pm 0.1 \mathrm{mg}$ (APHA 1994). Para estimar la biomasa de fitoplancton se determinó la concentración de clorofila $a$ $(\mu \mathrm{g} / \mathrm{L})$ a partir del filtrado de muestras subsuperficiales de volumen conocido, utilizando filtros de fibra de vidrio grado GF/F. Estos filtros se conservaron congelados hasta su procesamiento en laboratorio. La clorofila $a$ se extrajo de los filtros utilizando acetona al 90\% (se corrigió mediante cálculo de feopigmento acidificando el extracto), y se centrifugaron para el análisis final mediante método espectrofotométrico (Hauer and Lamberti 1996). La alcalinidad se midió en el campo por titulación con pH menor a 4.35.

\section{Muestreo de macrófitas acuáticas y plantas terrestres asociadas a mallines}

En cada una de las lagunas se establecieron tres transectas al azar de $15 \mathrm{~m}$ de longitud, que irradiaron desde el interior del cuerpo de 
agua hacia los bordes siguiendo alguno de los puntos cardinales o sus intermedios (S, N, E, O, $\mathrm{SE}, \mathrm{SO}, \mathrm{NE}, \mathrm{NO})$. A lo largo de cada transecta se ubicaron de manera sistemática 10 unidades muestrales circulares de $0.25 \mathrm{~m}^{2}$, separadas 1.5 m cada una (Figura 2). Debido a la variabilidad en las dimensiones de cada cuerpo de agua, en ocasiones, las unidades muestrales incluyeron sectores terrestres que fueron tratados de la misma manera que el resto de la transecta. En cada una de las unidades muestrales se registró la riqueza taxonómica y el porcentaje de cobertura por taxones. En todos los sitios se recolectaron tres ejemplares de las distintas macrófitas acuáticas/plantas terrestres presentes, se los colocó en bolsas plásticas rotuladas, se los herborizó y luego se los identificó en el laboratorio. Para identificarlos se utilizó un estereomicroscopio LEICA MZ6 y se consultaron claves y floras regionales, así como el catálogo de plantas vasculares del Cono Sur (Correa 1978-1999; Zuloaga et al. 2008). Cada ejemplar se determinó hasta el menor nivel taxonómico posible, y cada taxón se clasificó según su hábito $y$, de acuerdo con su origen, en nativo o exótico. En este último caso se consideraron las macrófitas en sentido estricto o hidrófitas considerando el hábito sumergido (incluidas las macroalgas de la división Charophyta), de hojas flotantes, y libre flotante/errante (U.S. EPA 2002; Chambers et al. 2008), y a las helófitas (plantas anfibias o típicas de humedales, adaptadas a períodos prolongados de anegamiento) (Kolada 2016; Svitok et al. 2018), clasificadas como emergentes en este trabajo. Además, siguiendo el criterio de Luque and Amari (1997), se incluyó a las plantas vasculares que

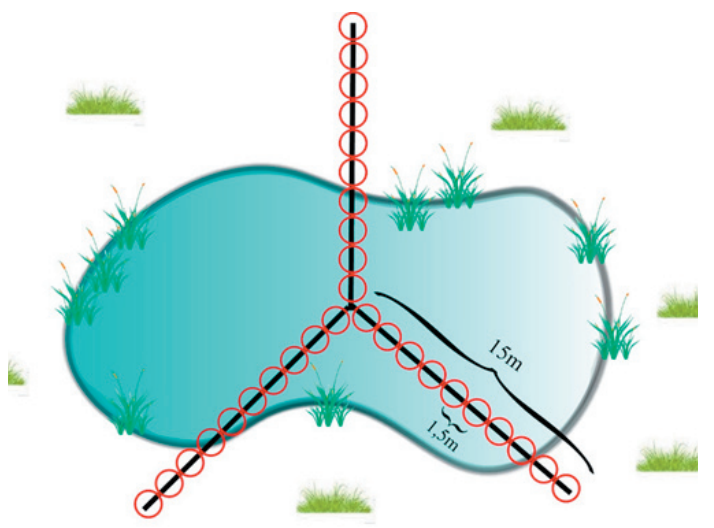

Figura 2. Croquis del diseño muestral realizado en cada sitio, en el cual se indican las transectas en color negro y las unidades muestrales circulares en rojo.

Figure 2. Sampling design conducted in each site, showing the transects in black and the circular sampling units in red. crecen en la matriz circundante inmediata al humedal en la categoría de terrestres.

\section{Análisis de datos}

Se analizaron las variables físicas y químicas de las lagunas para interpretar posibles patrones en las variables bióticas, es decir, relaciones entre taxones y ambiente. Con los datos de riqueza y abundancia de taxones se estimó la diversidad de Shannon-Wiener $\left(\mathrm{H}^{\prime}\right)$ para cada sitio. Para verificar la significancia de las diferencias entre los valores de riqueza y diversidad de las biozonas se realizaron análisis de varianza (ANOVA) de un factor, con un nivel de significancia de 0.05 . Se realizaron análisis multivariados de ordenamiento: 1) un ACP (análisis de componentes principales) para determinar la variabilidad de los sitios en función de las variables ambientales, y 2) un ACC (análisis de correspondencia canónica) para analizar la variabilidad de los taxones de macrófitas en los sitios en relación con las variables físicas y químicas. En el ACP se trabajó con una matriz de datos ambientales normalizados mediante el arcoseno de la raíz cuadrada para las variables medidas en porcentaje, y mediante el log10 $(\mathrm{X}+1)$ para el resto de las variables (exceptuando al $\mathrm{pH}$ ). El análisis se realizó en el software estadístico $\mathrm{R}$ versión 3.2.3 (R Development Core Team 2016), usando el paquete ade4 (función: dudi.pca; estandarizando variables por defecto) (Chessel et al. 2004). Aquellas variables que fueron consideradas redundantes fueron excluidas del análisis, observando el grado de correlación en el gráfico final. El ACC se basó en la matriz de abundancia de taxones y en la matriz de datos ambientales. Con el objetivo de alcanzar la normalidad, en ambos análisis, las variables correspondientes a la matriz ambiental fueron transformadas aplicándoles logaritmos $(\log 10[X+1])$. De igual manera, se aplicó el arcoseno de la raíz cuadrada a las variables de la matriz de abundancia de taxones. Se realizó una prueba de Monte Carlo para evaluar la consistencia de las relaciones obtenidas entre las variables (9999 iteraciones). Los análisis se realizaron utilizando el programa CANOCO (ter Braak and Smilauer 1998).

\section{Resultados}

\section{Descripción ambiental de los sitios}

Los valores medios de la totalidad de las variables físicas y químicas y ambientales 
Tabla 2. Valores medios ( \pm desvío estándar) de las variables físicas, químicas y climáticas para cada biozona analizada.

Table 2. Mean ( \pm standard deviation) of physical, chemical and climatic variables at each analyzed biozone.

\begin{tabular}{|c|c|c|c|}
\hline & Estepa & Ecotono & Bosque \\
\hline PMA (mm) & $227.3 \pm 64.06$ & $533.15 \pm 188.49$ & $754.56 \pm 224.02$ \\
\hline $\mathrm{T}^{\circ}$ agua $\left({ }^{\circ} \mathrm{C}\right)$ & $18.49 \pm 1.86$ & $19.46 \pm 4.92$ & $27.30 \pm 4.12$ \\
\hline Área $\left(\mathrm{m}^{2}\right)$ & $758.22 \pm 899.28$ & $5358.17 \pm 6424.93$ & $514.16 \pm 219.02$ \\
\hline Profundidad (m) & $0.65 \pm 0.58$ & $0.62 \pm 0.61$ & $0.34 \pm 0.19$ \\
\hline Conductividad $(\mu \mathrm{S} / \mathrm{cm})$ & $982.93 \pm 1632.42$ & $152.24 \pm 119.34$ & $169.54 \pm 172.44$ \\
\hline $\mathrm{TDS}(\mathrm{mg} / \mathrm{L})$ & $157.37 \pm 127.84$ & $82.26 \pm 64.82$ & $74.18 \pm 70.08$ \\
\hline Salinidad (\%o) & $0.50 \pm 1.02$ & $0.06 \pm 0.08$ & $0.02 \pm 0.04$ \\
\hline $\mathrm{O}_{2}(\mathrm{mg} / \mathrm{L})$ & $11.38 \pm 4.34$ & $12.20 \pm 0.89$ & $8.13 \pm 2.66$ \\
\hline $\mathrm{O}_{2}$ disuelto $(\%)$ & $128.44 \pm 48.52$ & $135.94 \pm 21.36$ & $105.9 \pm 36.4$ \\
\hline $\mathrm{pH}$ & $7.53 \pm 1.44$ & $7.04 \pm 1.15$ & $7.04 \pm 1.11$ \\
\hline $\mathrm{NT}(\mu \mathrm{g} / \mathrm{L})$ & $181.78 \pm 98.99$ & $190.04 \pm 83.86$ & $129.58 \pm 63.03$ \\
\hline $\mathrm{NO}_{3}+\mathrm{NO}_{2}(\mu \mathrm{g} / \mathrm{L})$ & $130.26 \pm 259.15$ & $566.56 \pm 1228.06$ & $31.6 \pm 27.3$ \\
\hline $\mathrm{NH}_{4}(\mu \mathrm{g} / \mathrm{L})$ & $40.93 \pm 22.32$ & $143.65 \pm 238.7$ & $94.38 \pm 102.45$ \\
\hline PT $(\mu \mathrm{g} / \mathrm{L})$ & $210.20 \pm 124.83$ & $218.58 \pm 228.95$ & $183.46 \pm 156.28$ \\
\hline $\operatorname{PRS}(\mu \mathrm{g} / \mathrm{L})$ & $123.29 \pm 127.08$ & $72.96 \pm 98.57$ & $113.67 \pm 110.53$ \\
\hline $\mathrm{NT}: \mathrm{PT}$ & $1.06 \pm 0.58$ & $2.36 \pm 2.41$ & $1.19 \pm 1.25$ \\
\hline TSS (mg/L) & $6.25 \pm 7.7$ & $171.46 \pm 404.36$ & $31.71 \pm 46.51$ \\
\hline Alcalinidad (Eq/L) & $3583.05 \pm 3095.39$ & $2043.98 \pm 1565.45$ & $2086.04 \pm 1678.95$ \\
\hline Clorofila $a(\mu \mathrm{g} / \mathrm{L})$ & $13.41 \pm 24.6$ & $26.59 \pm 39.64$ & $4.95 \pm 2.90$ \\
\hline
\end{tabular}

registradas en las biozonas se presentan en la Tabla 2. La superficie de las lagunas varió entre cuerpos de agua de unos pocos metros cuadrados hasta dos hectáreas. En cuanto a la profundidad, las lagunas de estepa presentaron mayores valores promedios en comparación a los de bosque (64 y $33 \mathrm{~cm}$, respectivamente). La conductividad varió entre $27.8 \mu \mathrm{S} / \mathrm{cm}$ y $4940 \mu \mathrm{S} / \mathrm{cm}$, siendo 488 $\mu \mathrm{S} / \mathrm{cm}$ el valor medio para la totalidad de los sitios. El valor obtenido del cociente NT/PT fue menor a 15 en todos los sitios, mientras que los valores de clorofila $a$ en general no superaron los $20 \mu \mathrm{g} / \mathrm{L}$. El valor promedio de oxígeno disuelto fue de $10.8 \mathrm{mg} / \mathrm{L}$ y el pH osciló entre 9.83 y 4.6 , siendo neutro' (próximo a 7 ) en la mayoría de los sitios.

Los primeros dos ejes del ACP capturaron el $43 \%$ de la variabilidad de los sitios en función de las características ambientales. El primer componente (ACP1) explicó el 24.8\% de variabilidad, y estuvo determinado por las siguientes variables: amonio, PT, PRS, total de sólidos en suspensión, alcalinidad, conductividad y clorofila $a$ (Figura 3a). El segundo componente (ACP2) capturó el $18.16 \%$ de variabilidad e involucró tanto variables físicas y climáticas (i.e., profundidad, temperatura del agua, precipitación media anual) como químicas (i.e., conductividad, $\mathrm{pH}$ y alcalinidad). En el ACP1, los sitios con concentraciones de nutrientes más elevadas (e.g., PT) y valores mayores de clorofila $a$ se agruparon hacia el extremo negativo, siendo éstos los de mayor nivel trófico. Por su parte, el ACP2 permitió separar los sitios en función de las biozonas, con las lagunas de estepa hacia el extremo negativo y las de bosque hacia el positivo (Figura 3b); la mayoría de los sitios de ecotono se ubicaron en la parte positiva del mismo.

\section{Caracterización de la comunidad de macrófitas}

Se registraron 62 taxones en total (ver Anexo), los cuales se repartieron en 59 angiospermas (30 monocotiledóneas y 29 dicotiledóneas), dos algas y una pteridófita. Las familias mejor representadas fueron Cyperaceae, Poaceae y Ranunculaceae con 12,8 y 6 taxones respectivamente. Los sitios que presentaron la mayor riqueza fueron PPA (estepa), con 12 taxones, y CHO (bosque) junto a RSG (estepa), con 11. Los valores medios de riqueza fueron levemente superiores en la estepa (8.62), en comparación con el bosque y el ecotono (7.4 y 6.85, respectivamente); estas diferencias no fueron estadísticamente significativas (F-radio $=0.695 ; \mathrm{P}=0.512$ ). Las especies más frecuentes fueron Myriophyllum quitense y Trifolium repens (registradas en 11 sitios), seguidas por Juncus balticus (registrada en 10 sitios). Del total de taxones relevados, 48 $(77.5 \%)$ resultaron nativos y los 12 restantes (22.5\%) fueron exóticos (ver Anexo). Las lagunas de bosque tuvieron el mayor porcentaje de macrófitas exóticas (29\%), 
a)

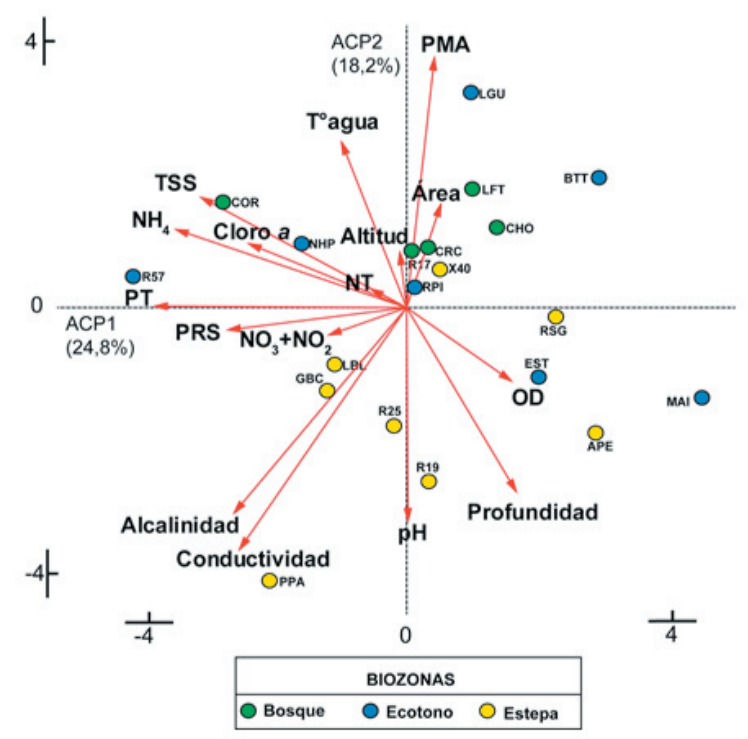

b)

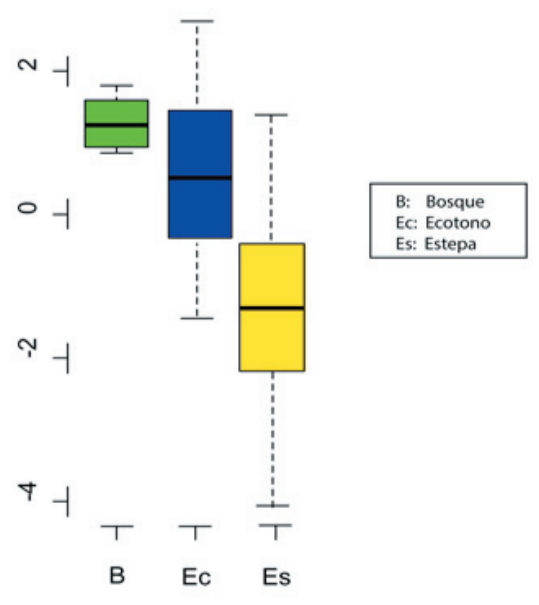

Figura 3. a) Análisis de componentes principales que determina las agrupaciones de los sitios muestreados en función de variables ambientales medidas en 20 lagunas de la provincia de Chubut (los códigos se consignan en la Tabla 1). b) Boxplot del eje ACP2 en función de las biozonas. PRS: fósforo reactivo soluble; TSS: total de sólidos en suspensión; PMA: precipitación media anual; PT: fósforo total; NT: nitrógeno total; cloro $a$ : clorofila $a$.

Figure 3. a) Principal component analysis of sites and environmental variables measured at 20 shallow lakes in the province of Chubut (full names of sites codes are given in Table 1). b) Boxplot of the PCA2 axis according to the biozones. PRS: soluble reactive phosphorus; TSS: total suspended solids; PMA: mean annual precipitation; PT: total phosphorus; NT: total nitrogen; cloro $a$ : chlorophyll $a$.

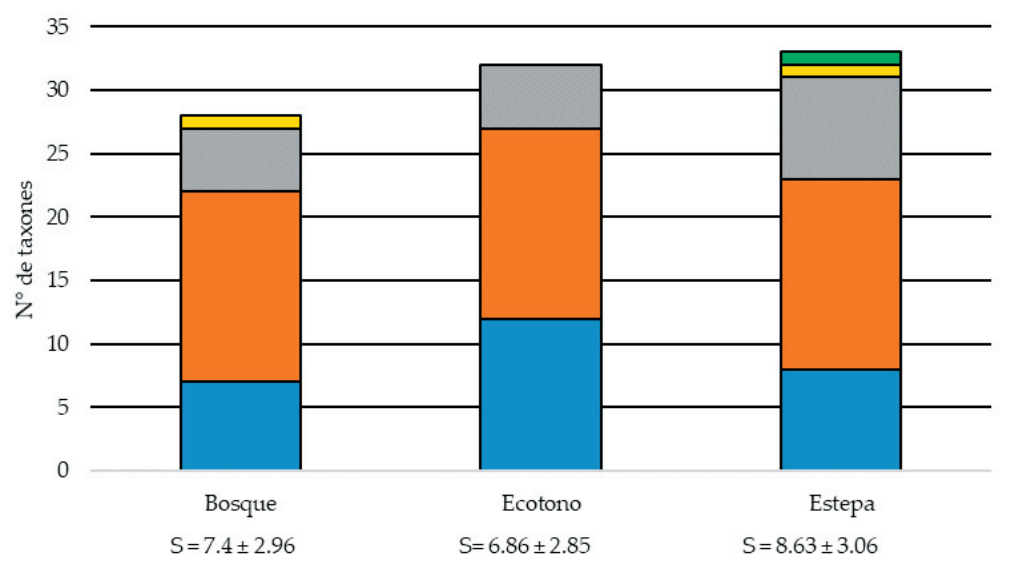

Figura 4. Riqueza de especies en función de la biozona, discriminada según el hábito de vida. S: valor medio $( \pm$ desvío estándar) de riqueza de especies.

Figure 4. Species richness from each biozone. Colors are used to distinguish among life habits. S: mean species richness value $( \pm$ standard deviation). mientras que la estepa presentó el mayor porcentaje de nativas, con un 85\% (Tabla 3).

En cuanto a los hábitos de vida, dominaron las macrófitas emergentes con 28 representantes $(45 \%)$, seguidos por las sumergidas con 9 taxones (15\%); las macrófitas arraigadas de hojas flotantes y las libremente flotantes fueron representadas con una sola especie cada uno (Potamogeton linguatus y Lemna gibba, respectivamente) (2\%). El resto del elenco de plantas fue terrestre (23 taxones), y compuesto casi exclusivamente por poáceas. Las lagunas de ecotono tuvieron la mayor cantidad de especies terrestres asociadas a los mallines (12 taxones); las macrófitas emergentes se vieron representadas equitativamente en las tres biozonas (15 taxones en cada una), mientras que las lagunas de estepa presentaron la mayor cantidad de taxones con hábitos sumergidos (8) (Figura 4).

Los datos referidos a los atributos de la comunidad de macrófitas evaluados en 
Tabla 3. Atributos de la comunidad de macrófitas que caracterizan a las lagunas de mallines ubicados en las distintas biozonas. Los porcentajes que superan el $100 \%$ consideran la cobertura de flotantes más sumergidas.

Table 3. Macrophyte community metrics that characterize the shallow lakes from different biozones. The percentages that exceed the $100 \%$ consider the addition of submerged and floating species coverage.

\begin{tabular}{|c|c|c|c|c|c|c|c|c|c|c|}
\hline 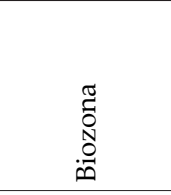 & $\frac{\Xi}{\sum}$ & 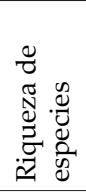 & $\begin{array}{l}\widetilde{0} \\
\stackrel{0}{0} \\
0 \\
0 \\
0 \\
0\end{array}$ & I & 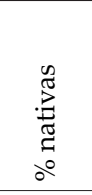 & 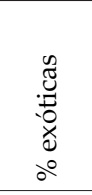 & 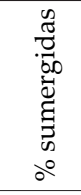 & 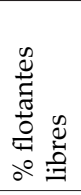 & 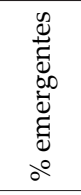 & 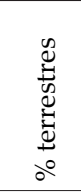 \\
\hline \multirow[t]{5}{*}{ Bosque } & $\mathrm{CHO}$ & 11 & 137.64 & 1.85 & 100 & 0 & 25 & 12.5 & 50 & 12.5 \\
\hline & COR & 6 & 48.48 & 1.18 & 50 & 50 & 0 & 0 & 33.3 & 66.7 \\
\hline & R17 & 4 & 11.15 & 1.31 & 100 & 0 & 25 & 0 & 75 & 0 \\
\hline & CRC & 6 & 116.49 & 1.19 & 83.3 & 16.7 & 16.7 & 0 & 66.6 & 16.7 \\
\hline & LFT & 10 & 73.62 & 1.15 & 80 & 20 & 20 & 0 & 50 & 30 \\
\hline Valor medio & & 7.4 & 77.48 & 1.34 & 82.66 & 17.34 & 17.34 & 3.13 & 54.98 & 25.18 \\
\hline \multirow[t]{7}{*}{ Ecotono } & MAI & 10 & 46.57 & 1.39 & 60 & 40 & 10 & 0 & 50 & 40 \\
\hline & BTT & 5 & 95.65 & 1.06 & 100 & 0 & 40 & 0 & 40 & 20 \\
\hline & LGU & 4 & 83.65 & 0.38 & 100 & 0 & 0 & 0 & 25 & 75 \\
\hline & RPI & 8 & 73.48 & 1.53 & 75 & 25 & 25 & 0 & 37.5 & 37.5 \\
\hline & R57 & 3 & 1.32 & 1.04 & 66.7 & 33.3 & 0 & 0 & 33.3 & 66.7 \\
\hline & EST & 10 & 35.79 & 1.5 & 70 & 30 & 30 & 0 & 30 & 40 \\
\hline & NHP & 8 & 64.47 & 1.01 & 85.7 & 14.3 & 28.6 & 0 & 42.8 & 28.6 \\
\hline Valor medio & & 6.86 & 57.28 & 1.13 & 79.63 & 20.37 & 19.09 & 0 & 36.94 & 43.97 \\
\hline \multirow[t]{8}{*}{ Estepa } & PPA & 12 & 75.5 & 1.95 & 91.7 & 8.3 & 16.7 & 8.3 & 41.7 & 33.3 \\
\hline & APE & 3 & 35.7 & 0.62 & 100 & 0 & 50 & 0 & 0 & 50 \\
\hline & R25 & 10 & 89.95 & 1.71 & 88.9 & 11.1 & 22.2 & 0 & 33.3 & 44.5 \\
\hline & R19 & 10 & 78.28 & 1.38 & 80 & 20 & 20 & 0 & 50 & 30 \\
\hline & GBC & 5 & 95.31 & 0.97 & 100 & 0 & 20 & 20 & 60 & 0 \\
\hline & X40 & 9 & 66.97 & 1.63 & 88.9 & 11.1 & 44.5 & 0 & 33.3 & 22.2 \\
\hline & RSG & 11 & 63.11 & 1.91 & 72.7 & 27.3 & 18.1 & 9.1 & 36.4 & 36.4 \\
\hline & LBL & 9 & 70.8 & 1.28 & 66.7 & 33.3 & 33.3 & 0 & 44.5 & 22.2 \\
\hline Valor medio & & 8.63 & 71.95 & 1.43 & 86.11 & 13.89 & 28.1 & 4.68 & 37.40 & 29.83 \\
\hline
\end{tabular}

$\stackrel{ }{\circ}$

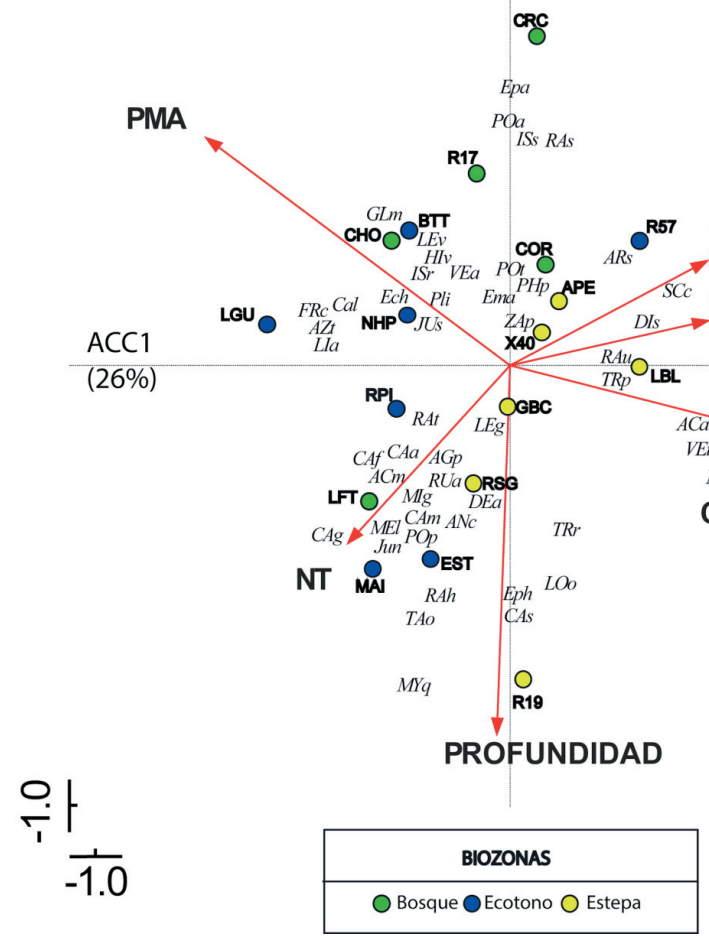

Figura 5. Análisis de correspondencia canónica que relaciona la abundancia de los 62 taxones relevados y los parámetros ambientales de los 20 mallines de la provincia de Chubut, respectivamente (ver códigos en el Anexo y en la Tabla 1). PRS: fósforo reactivo soluble; NT: nitrógeno total; PMA: precipitación media anual.

Figure 5. Canonical correspondence analysis based on the abundance of the 62 macrophyte taxa and environmental parametersat 20 mallines of the province of Chubut, respectively (full names of sites and taxa codes are given in Appendix and Table 1). PRS: soluble reactive phosphorus; NT: total nitrogen; PMA: mean annual precipitation. 
cada una de las 20 lagunas se sintetizan en la Tabla 3. Las lagunas de estepa presentaron un valor levemente superior de diversidad $\left(\mathrm{H}^{\prime}=1.43\right)$, en comparación con las de bosque y ecotono $\left(\mathrm{H}^{\prime}=1.34\right.$ y 1.13 , respectivamente); $\sin$ embargo, estas diferencias tampoco resultaron significativas (F-radio=1.048; $\mathrm{P}=0.372$ ).

\section{Relación entre variables ambientales y ensambles de macrófitas}

En los resultados del ACC (Figura 5), se observa que el ACC1 (26\% de la variabilidad) explica un gradiente ambiental físico y químico, con las variables conductividad y PMA como principales determinantes. E1 ensamble de especies asociadas a la biozona bosque estuvo compuesto por Glyceria multiflora, Legenere valdiviana, Hippuris vulgaris e Isolepis ranko. En las lagunas de estepa las especies más frecuentes fueron Eleocharis nubigena, Poa lanuginosa, Grindelia chiloensis y Stuckenia filiformis. La especie que mejor caracterizó a los sitios más profundos fue Myriophyllum quitense. Por otra parte, el ensamble de macrófitas compuesto por Arenaria serpens, Schoenoplectus californicus y Distichlis spicata, fue el que mejor toleró sitios con $\mathrm{pH}$ alcalino y gran contenido de PRS. La prueba de Monte Carlo corroboró las relaciones establecidas en el análisis, con valores de probabilidad significativos tanto para el primer eje (F-radio=1.307; $\mathrm{P}=0.033$ ) como para la suma de todos los canónicos (F-radio=1.176; $\mathrm{P}=0.029$ ).

\section{Discusión}

Las lagunas de mallines de estepa, asociados a escasas precipitaciones (Chimner et al. 2011; Epele et al. 2018) se caracterizaron por mostrar los mayores valores de conductividad, $\mathrm{pH}$ y concentraciones de nutrientes (PRS, PT y NT). Sin embargo, en general, los sitios se mantuvieron en rangos moderados de conductividad (el 90\%, entre 50 y $1000 \mu \mathrm{S} / \mathrm{cm}$ ) y nutrientes, en relación con lo descrito en trabajos comparables de la Patagonia (Perotti et al. 2004; Epele and Miserendino 2015). El rango de valores de $\mathrm{pH}$ se encuentra dentro de los límites de tolerancia para la supervivencia de la mayoría de los organismos acuáticos (i.e., 4-11) (Conde and Gorga 1999). El promedio del porcentaje de saturación de oxígeno disuelto en las lagunas de estepa (128.4\%) fue mayor al de los sitios de bosque (105.9\%), lo que podría deberse a los fuertes y constantes vientos del oeste (Perotti et al. 2005), pero también a la fotosíntesis de las macrófitas que, justamente, fueron abundantes en esta biozona.

Tres de las lagunas de estepa (sitios R19, GBC y LGB) presentaron valores de PT y clorofila a que permiten clasificarlas como eutróficas (i.e., PT entre 25 y $100 \mu \mathrm{g} / \mathrm{L}$ y clorofila $a$ entre 7 y 40 g/L) (OECD 1982; Moreno Franco et al. 2010). Sin embargo, y en base a lo reportado por Epele y colaboradores (2018), en un estudio de 109 mallines distribuidos en toda la Patagonia, utilizar el PT como parámetro del nivel trófico de estas lagunas podría llevar a interpretaciones erróneas, ya que el mismo se encontraría naturalmente en exceso en estos ecosistemas. En general, las macrófitas tienden a ser más susceptibles a los cambios de nitrógeno que a los de fósforo; este hecho se evidencia en los valores de cobertura en sus hábitats (Søndergaard et al. 2017), y toma más relevancia al tener en cuenta la elevada evapotranspiración de la estepa (del Valle et al. 1998), que en épocas estivales provocaría una disminución del volumen de agua de las lagunas y la consecuente concentración de sales y nutrientes. Estos valores no llegarían a influir en los niveles de fitoplancton, por lo que tampoco alterarían las concentraciones de oxígeno disuelto esencial para la presencia de macrófitas (Roldán and Ramírez 2008; Moreno Franco et al. 2010). Sin embargo, en esta biozona, los efectos de la ganadería, del cambio climático (i.e., aumento de temperatura y disminución de precipitaciones) y de la radiación ultravioleta podrían acentuar la eutrofización en las lagunas (Brönmark and Hansson 2002).

Las familias mejor representadas en este trabajo coincidieron con lo reportado por otros estudios en la Patagonia (Kutschker et al. 2014; Jocou et al. 2018; Manzo et al. 2020), los que también indicaron a las familias Poaceae y Cyperaceae como dominantes en mallines. El alto esfuerzo de muestreo de este estudio quedó reflejado en los valores de riqueza de especies hallados, siendo la relación sitios/especies superior a la que reportaron estudios similares en la Patagonia (Kutschker et al. 2014). Sin embargo, esto también se podría explicar por la inclusión en el presente estudio de especies terrestres asociadas a los humedales. Por otra parte, en contraste con lo registrado por Macchi (2017), tanto la diversidad como el valor medio de riqueza de especies fueron levemente superiores para la estepa respecto al bosque. Nuevamente, esto podría deberse al hecho de que las lagunas de estepa contienen en sus márgenes, 
además de la comunidad de macrófitas, una gran variedad de especies típicas de mallín y terrestres asociadas, que la metodología de este trabajo permitió representar, ya que las transectas abarcaron gran parte del gradiente de humedad. En cambio, tanto el bosque como el ecotono poseen márgenes dominados casi exclusivamente por macrófitas emergentes. Este tipo de muestreo cuantitativo y estratificado se propone como una técnica adecuada para abarcar el amplio gradiente de humedad que suele haber en estos ecosistemas, pudiendo detectar así diferencias leves. También se podría implementar para evaluar gradientes de impacto antrópico.

En la estepa se registraron todas las formas de vida (i.e., emergentes, sumergidas, flotantes libres y de hojas flotantes), probablemente porque los espejos de agua en esta zona muestran total exposición a la luz; esto estimula la actividad primaria de los organismos fotosintéticos (Osborne 2012), a diferencia de lo que ocurre en el ecotono y el bosque, en donde la vegetación arbustiva y la arbórea de las inmediaciones puede disminuir la incidencia lumínica en el cuerpo de agua (León et al. 1998). Por otra parte, el hecho de que la estepa contenga un mayor número de especies nativas en comparación con el bosque, donde se encuentran mayor cantidad de exóticas, se podría explicar por la facilidad que tiene la vegetación invasora para colonizar y desarrollarse en ambientes con mayor humedad (Cuassolo et al. 2012; Urrutia et al. 2017).

En la totalidad de los sitios de estepa con mayores concentraciones de nutrientes, las macrófitas emergentes fueron las que presentaron los mayores valores de densidad. Según Brix (1994) la capacidad absorción de fósforo por parte de las macrófitas emergentes es superior al de las sumergidas, lo cual explicaría el hecho de que los sitios con los mayores valores de PT hayan presentado mayor abundancia de macrófitas emergentes, en comparación con las que se encuentran bajo la superficie del agua. Esta relación coincide con lo descripto por Manzo y colaboradores (2020) para la comunidad de macrófitas presente en lagunas someras de las provincias de Santa Cruz y Tierra del Fuego. Rogers y colaboradores (1985) también demostraron esta relación en humedales de Sudáfrica destinados al tratamiento de aguas residuales. Por otra parte, en estos ambientes son los ensambles de macroalgas los que reemplazan a la mayoría de las macrófitas (Sayer et al. 2010), ya que gracias sus altas tasas de renovación reflejan y responden rápidamente a los procesos de eutrofización. Era de esperarse entonces, que en estos sitios se concentrara la mayor proporción de los taxones Nitella sp. y Chara sp. Por esta razón, es probable que las macrófitas sumergidas en ambientes de este tipo se vean perjudicadas, y sean las emergentes las que aprovechan esta situación al no competir directamente con las macroalgas por la oferta de nutrientes y luz.

En función de las variables ambientales y los atributos de las comunidades de macrófitas analizados, se podría establecer un gradiente desde los mallines ubicados en las biozonas de bosque y ecotono cuyas características resultaron similares, hasta los mallines de estepa que presentaron comunidades adaptadas a dicho ambiente. Leves fluctuaciones en los niveles de precipitación pueden afectar la integridad de los humedales (Burkett and Kusler 2000). En consecuencia, la biodiversidad de macrófitas de lagunas temporarias (típicas de estepa) se podría ver afectada severamente en el escenario del cambio climático (Crego et al. 2013). Además, si bien todos los sitios aquí estudiados no estaban afectados de manera intensiva por actividades antrópicas, se debería tener en cuenta que el uso ganadero, sumado a los mencionados efectos adversos del cambio climático, podrían generar alteraciones considerables sobre estos ecosistemas y poner en peligro su integridad. Este estudio se debería complementar con muestreos a largo plazo para evidenciar la evolución en el tiempo de estos ambientes, comparando, además, sitios sometidos a disturbios de origen antrópico con sitios de referencia. Los resultados de este trabajo podrían utilizarse como herramienta para monitorear y bioindicar la calidad del agua de los mallines, a partir de las asociaciones de macrófitas típicas de cada biozona.

Agradecimientos. Queremos agradecer a la Dra. L. Acheritobehere por su colaboración en la confección del mapa de curvas pluviométricas, y al Dr. P. López Bernal quien asesoró en el diseño muestral y análisis estadístico. El presente estudio se llevó a cabo en el marco del proyecto "Biodiversidad de invertebrados acuáticos en ambientes asociados a mallines de Patagonia, con especial énfasis en culícidos" (Res: R/8 N 108-2013). 


\section{REFERENCIAS}

American Public Health Association (APHA). 1992. Standard methods for the examination of water and wastewater. American Water Works Association, Hanover, Maryland, USA.

American Public Health Association (APHA). 1994. Standard methods for the examination of water and wastewater. American Public Health Association, Washington DC, USA.

Balcombe, C., K. Anderson, J. T. Fortney, R. H. Rentch, J. S. Grafton, W. S. Kordek, and W. Virginia. 2005. A comparison of plant communities in mitigation and reference wetlands in the mid-appalachians. Wetlands 25(1):130-142. https: //doi.org/10.1672/0277-5212(2005)025[0130:ACOPCI]2.0.CO;2.

Brix, H. 1994. Functions of macrophytes in constructed wetlands. Water Science and Technology 29(4):71-78. https: //doi.org/10.2166/wst.1994.0160.

Brönmark, C., and L. A. Hansson. 2002. Environmental cues in lakes and ponds: current state and perspectives. Environmental Conservation 29:290-306. https://doi.org/10.1017/S0376892902000218.

Crego, R. D., C. K. Nielsen, and K. A. Didier. 2013. Climate change and conservation implications for wet meadows in dry Patagonia. Environmental Conservation 41(2):122-131. https://doi.org/10.1017/s037689291300026x.

Burkett, V., and J. Kusler. 2000. Climate change: potential impacts and interactions in wetlands of the United States. Journal of the American Water Resources Association 36(2):313-320. https://doi.org/10.1111/j.1752-1688.2000.tb04270.x.

Carpenter, S. R., and D. M. Lodge. 1986. Effects of submersed macrophytes on ecosystem processes. Aquatic Botany 26:341-370. https://doi.org/10.1016/0304-3770(86)90031-8.

Chambers, P. A., P. Lacoul, K. J. Murphy and S. M. Thomaz. 2008. Global diversity of aquatic macrophytes in freshwater. Hydrobiologia 595(1):9-26. https://doi.org/10.1007/s10750-007-9154-6.

Chessel, D., A. B. Dufour, and J. Thioulouse. 2004. The ade4 package-I-One-table methods. R News 4:5-10.

Chimner, R. A., G. L. Bonvissuto, M. V. Cremona, J. J. Gaitán, and C. R. López. 2011. Ecohydrological conditions of wetlands along a precipitation gradient in Patagonia, Argentina. Ecología Austral 21:329-337.

Collantes, M. B., and A. M. Faggi. 1999. Los humedales del sur de Sudamérica. Pp. 15-25 en A. I. Málvarez (ed.). Tópicos sobre humedales subtropicales y templados de Sudamérica. Unesco, Montevideo, Uruguay.

Conde, D., and J. Gorga. 1999. Composición Iónica. Pp. 65-77 en R. Arocena and D. Conde (eds.). Métodos en ecología de aguas continentales, con ejemplos de limnología en Uruguay. DIRAC, Montevideo, Uruguay.

Correa, M. O. 1978-1999. Flora Patagónica. Tomo VIII: parte I, II, III, IVa, IVb, V, y VIII. Colección científica del INTA. Buenos Aires.

Cuassolo, F., E. Balseiro, and B. Modenutti. 2012. Alien vs. native plants in a Patagonian wetland: Elemental ratios and ecosystem stoichiometric impacts. Biological Invasions 14(1):179-189. https://doi.org/10.1007/s10530-011-9995-9.

Epele, L. B., M. G. Grech, L. M. Manzo, P. A. Macchi, V. Hermoso, M. L. Miserendino, N. Bonada, and M. CañedoArgüelles. 2021. Identifying high priority conservation areas for Patagonian wetlands biodiversity. Biodiversity and Conservation 30:1359-1374. https://doi.org/10.1007/s10531-021-02146-2.

Epele, L. B., L. Manzo, M. Grech, P. Macchi, A. Ñ. Claverie, L. Lagomarsino, and M. L. Miserendino. 2018. Disentangling natural and anthropogenic influences on Patagonian pond water quality. Science of the Total Environment 613-614: 866-876. https://doi.org/10.1016/j.scitotenv.2017.09.147.

Epele, L. B., and M. L. Miserendino. 2015. Temporal dynamics of invertebrate and aquatic plant communities at three intermittent ponds in livestock grazed Patagonian wetlands grazed Patagonian wetlands. Journal of Natural History 50:711-730. https://doi.org/10.1080/00222933.2015.1062930

Erize, E. 1960. Diccionario comentado Mapuche-Español. Araucano, Pehuenche, Pampa, Picunche, Rancülhue, Huilliche. Editorial Peuser y Cuadernos del Sur, Buenos Aires, Argentina.

Gaitán, J. J., C. R. López, and D. Bran. 2011. Vegetation composition and its relationship with the environment in mallines of north Patagonia, Argentina. Wetlands Ecology Manage 19:121-130. https://doi.org/10.1007/s11273-010-9205-z.

Gaitán, J. J., D. Bran, G. Oliva, F. T. Maestre, and M. R. Aguiar. 2014. Plant species richness and shrub cover attenuate drought effects on ecosystem functioning across Patagonian rangelands. Biology Letters 10:1020140673. https: //doi.org/10.1098/rsbl.2014.0673.

Gosselink, J. G., and E. Turner. 1978. The role of hydrology in fresh water wetland ecosystems. Pp. 63-78 en R. E. Good, D. F. Whigham and R. L. Simpson (eds.). Freshwater Wetlands: Ecological Processes and Management Potential. Academic Press, New York, USA.

Hauenstein, E., M. Gonzáles, F. Peña-Cortez and A. Muñoz-Pedreros. 2002. Clasificación y caracterización de la flora y vegetación de los humedales de la costa de Toltén (IX Región, Chile). Gayana Botánica 59:87-100. https://doi.org/ 10.4067/S0717-66432002000200006.

Hauer, F. R., and G. A. Lamberti. 1996. Methods in stream ecology. Academic Press. New York, USA.

Hauser, A. 1984. Mallines: Caracterización geológica, geomorfológica y geotécnica; métodos constructivos utilizados en el camino longitudinal austral para sobrepasarlos. Revista Geológica de Chile 22:77-89.

Iriondo, M. 1989. Quaternary lakes of Argentina. Palaeogeography, Palaeoclimatology and Palaeoecology 70:81-88. https://doi.org/10.1016/0031-0182(89)90081-3.

Jocou, A. I., C. Fernández, and R. Gandullo. 2018. Macrófitas acuáticas vasculares del sistema de drenaje del Alto Valle de Río Negro, Patagonia (Argentina). Revista del Museo de La Plata 3:296-308. https://doi.org/10.24215/25456377e060.

Junk, W. J., P. B. Bayley and R. E. Sparks. 1989. The flood pulse concept in river-floodplain systems. Canadian special 
publication of fisheries and aquatic sciences 106(1):110-127.

Kolada, A. 2016. The use of helophytes in assessing eutrophication of temperate lowland lakes: Added value? Aquatic Botany 129:44-54. https://doi.org/10.1016/j.aquabot.2015.12.002.

Kutschker, A., L. B. Epele, and M. L. Miserendino. 2014. Aquatic plant biodiversity and environmental relationships in grazed northwest Patagonian wetlands, Argentina. Ecological Engineering 64:37-48. https://doi.org/10.1016/ j.ecoleng.2013.12.007.

León, R. C., D. Bran, M. Collantes, J. M. Paruelo, and A. Soriano. 1998. Grandes unidades de vegetación de la Patagonia extra andina. Ecología Austral 8:125-144.

Luque, J. L., and M. Amari. 1997. Características edáficas e hídricas de los mallines del Chubut. Estación experimental agropecuaria INTA. Laboratorio de análisis agronómicos. Trelew, Chubut, Argentina.

Macchi, P. A. 2017. Macroinvertebrados acuáticos como indicadores de cambios en el uso del suelo en mallines del sudoeste de la provincia de Río Negro. Tesis de doctorado. Facultad de Ciencias Naturales y Museo. Universidad Nacional de La Plata, Buenos Aires. Argentina. Pp. 227.

Manzo, L. M., M. G. Grech, L. B. Epele, A. M. Kutschker, and M. L. Miserendino. 2020. Macrophyte regional patterns, metrics assessment and ecological integrity of isolated ponds at Austral Patagonia (Argentina). Science of The Total Environment 727:138617. https://doi.org/10.1016/j.scitotenv.2020.138617

Mitsch, W. J., and J. G. Gosselink. 2015. Wetlands, 5th edn. John Wiley and Sons, Hoboken, New Jersey, USA.

Moreno Franco, D., J. Quintero, and A. López. 2010. Métodos para identificar, diagnosticar y evaluar el grado de eutrofia. Revista Contactos 78:25-33.

Mushet, D. M., N. H. Euliss Jr., and T. L. Shaffer. 2002. Floristic quality assessment of one natural and three restored wetland complexes in North Dakota, USA. Wetlands 22(1):126-138. https://doi.org/10.1672/0277-5212(2002)022[0126: FQAOON]2.0.CO;2.

OECD. 1982. Eutrophication of waters. Monitoring, assessment and control. OECD, Paris, Francia.

Osborne, L.R. 2012. Phosphorus Immobilization of Lead in Wetland Soils of the Coeur D'Alene River Basin, Idaho. Doctoral Thesis. University of Idaho, Moscow, Idaho, USA.

Paruelo, J. M., R. A. Golluscio, J. P. Guerschman, A. Cesa, V. V. Jouve, and M. F. Garbulsky. 2004. Regional scale relationships between ecosystem structure and functioning: the case of the Patagonian steppes. Global, Ecology and Biogeography 13:385-395. https://doi.org/10.1111/j.1466-822X.2004.00118.x.

Paruelo, J. M., A. Beltrán, E. Jobbágy, O. E Sala, and R. A. Golluscio. 1998. The climate of Patagonia: general patterns and controls on biotic processes. Ecología Austral 8:85-101.

Perotti, M. G., C. Diéguez, P. Pérez, and F. Jara. 2004. Consideraciones sobre el efecto de las variables del clima y las interacciones biológicas sobre las comunidades acuáticas de humedales patagónicos. Actas del taller: Los Mallines en la Patagonia Argentina. Volumen 4. INTA, Ciefap, Esquel, Chubut, Argentina.

Perotti, M. G., M. C. Diéguez, and F. Jara. 2005. Estado del conocimiento de humedales del norte patagónico (Argentina): aspectos relevantes e importancia para la conservación de la biodiversidad regional. Revista Chilena de Historia Natural 78:723-737. https://doi.org/10.4067/S0716-078X2005000400011.

R Development Core Team. 2016. R: A Language and Environment for Statistical Computing. R Version 3.2.3. R Foundation for Statistical Computing, Vienna, Austria.

Raffaele, E. 1999. Mallines: Aspectos generales y problemas particulares. Pp. 27-34 en A. I. Malvárez (ed.). Tópicos sobre humedales subtropicales y templados de Sudamérica. UNESCO, Montevideo, Uruguay.

Rogers, F. E. J., K. H. Rogers, and J. S. Buzer. 1985. Wetlands for Wastewater Treatment with Special Reference to Municipal. Wastewaters. Witwatersrand University Press, Johannesburg, South Africa.

Roldán, P. G., and J. J. Ramírez. 2008. Fundamentos de limnología neotropical, º edición . Editorial Universidad de Antioquia, Medellín, Colombia.

San Martín, C., Y. Pérez, M. Álvarez, C. Salazar, and C. Ramírez. 2013. Diversidad vegetal de lagunas temporales en la estepa patagónica chilena noroccidental. Anales Instituto Patagonia 41:111-116. https://doi.org/10.4067/S0718686X2013000200012.

Sayer, C. D., T. A. Davidson, and J. I. Jones. 2010. Seasonal dynamics of macrophytes and phytoplankton in shallow lakes: a eutrophication-driven pathway from plants to plankton? Freshwater Biology 55(3):500-513. https://doi.org/ 10.1111/j.1365-2427.2009.02365.x.

Secretaría de la Convención de Ramsar. 2006. Manual de la Convención de Ramsar: Guía a la Convención sobre los Humedales (Ramsar, Irán, 1971). $4^{\circ}$ edición. Secretaría de la Convención de Ramsar. Gland, Suiza.

Svitok, M., M. Novikmec, L. Hamerlík, J. Kochjarová, H. Otahelóvá, P. Palove-Balang, D. Senko, Z. Matúsová, K. Buvíková, and R. Hrivnák. 2018. Test of the efficiency of environmental surrogates for the conservation prioritization of ponds based on macrophytes. Ecological Indicators 95(1):606-614. https://doi.org/10.1016/j.ecolind.2018.08.006.

Søndergaard, M., T. L. Lauridsen, L. S. Johansson, and E. Jeppesen. 2017. Nitrogen or phosphorus limitation in lakes and its impact on phytoplankton biomass and submerged macrophyte cover. Hydrobiologia 795(1):35-48. https: //doi.org/10.1007/s10750-017-3110-x.

ter Braak, C. J. F., and P. Smilauer. 1998. CANOCO Reference manual and User's guide t Canoco for Windows: software for Canonical Community Ordination (version 4). Microcomputer Power. Ithaca, New York, USA.

Thomaz, S. M., and E. R. Cunha. 2010. The role of macrophytes in habitat structuring in aquatic ecosystems: methods of measurement, causes and consequences on animal assemblages' composition and biodiversity. Acta Limnologica 
Brasiliensia 22(02):218-236. https://doi.org/10.4322/actalb.02202011

Thomaz, S. M., F. A. Lansac-Tôha, M. C. Roberto, F. A. Esteves, and A. F Lima. 1992. Seasonal variation of some limnological features of lagoa do Guaraná, a várzea lake of the High Rio Paraná, State of Mato Grosso do Sul, Brazil. Revue d'Hydrobiolgie Tropicale 25(4):269-276.

Urrutia, J., P. Sánchez, A. Pauchard, and E. Hauenstein. 2017. Invasive aquatic plants presents in Chile: Distribution, traits of life and invasive potential. Gayana - Botanica 74:147-157. https://doi.org/10.4067/S0717-66432017005000324.

U.S. Environmental Protection Agency. 2002. Health assessment document for diesel engine exhaust. National Center for Environmental Assessment, Washington DC, USA.

del Valle, H. F., N. O. Elissalde, D. A. Gagliardini, and J. Milovich. 1998. Status of desertification in the Patagonian region: Assessment and mapping from satellite imagery. Arid Soil Research and Rehabilitation 12:1-27. https: //doi.org/10.1080/15324989809381502.

Zuloaga, F., O. Morrone, and M. Belgrano. 2008. Catálogo de las plantas vasculares del Cono Sur. Monographs in Systematic Botany from the Missouri Botanical Garden 107:1-3348. 